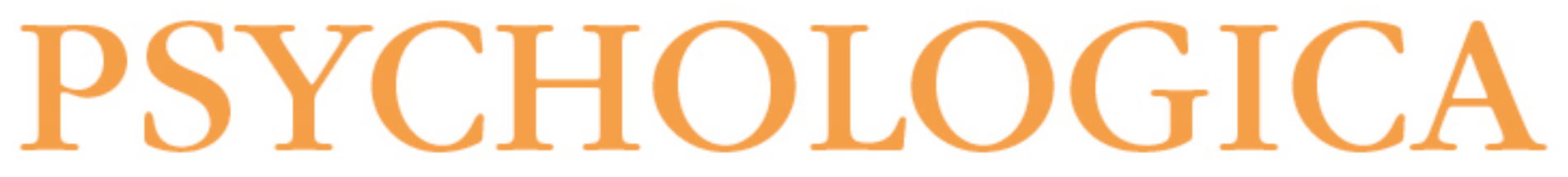

Efeitos da orientação para a aprendizagem e da segurança psicológica nos conflitos nas equipas

Autor(es): $\quad$ Passos, Ana Margarida; Silva, Sílvia A.; Santos, Catarina M.

Publicado por: Imprensa da Universidade de Coimbra

URL

persistente: URI:http://hdl.handle.net/10316.2/5599

Accessed : $\quad$ 26-Apr-2023 12:59:19

A navegação consulta e descarregamento dos títulos inseridos nas Bibliotecas Digitais UC Digitalis, UC Pombalina e UC Impactum, pressupõem a aceitação plena e sem reservas dos Termos e Condições de Uso destas Bibliotecas Digitais, disponíveis em https://digitalis.uc.pt/pt-pt/termos.

Conforme exposto nos referidos Termos e Condições de Uso, o descarregamento de títulos de acesso restrito requer uma licença válida de autorização devendo o utilizador aceder ao(s) documento(s) a partir de um endereço de IP da instituição detentora da supramencionada licença.

Ao utilizador é apenas permitido o descarregamento para uso pessoal, pelo que o emprego do(s) título(s) descarregado(s) para outro fim, designadamente comercial, carece de autorização do respetivo autor ou editor da obra.

Na medida em que todas as obras da UC Digitalis se encontram protegidas pelo Código do Direito de Autor e Direitos Conexos e demais legislação aplicável, toda a cópia, parcial ou total, deste documento, nos casos em que é legalmente admitida, deverá conter ou fazer-se acompanhar por este aviso. 


\section{NÚMERO 55}

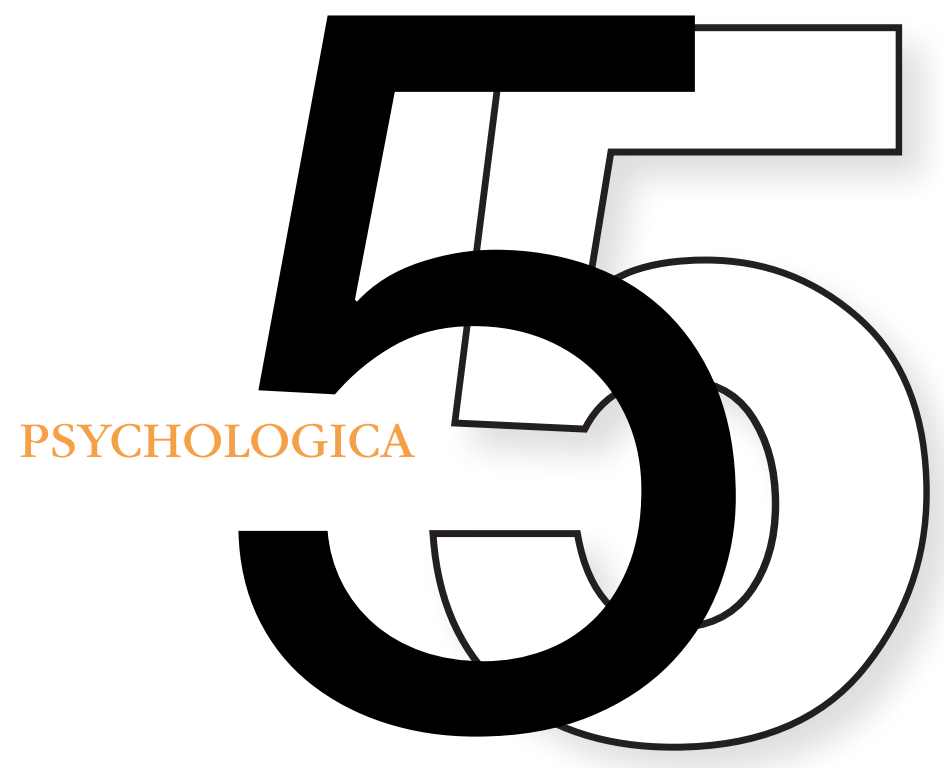

PSICOLOGIA DAS

ORGANIZAÇÕES

DO TRABALHO E DOS

RECURSOS HUMANOS 


\title{
Efeitos da orientação para a aprendizagem e da segurança psicológica nos conflitos nas equipas
}

\author{
Ana Margarida Passos ${ }^{1}$, Sílvia A. Silva² \& Catarina M. Santos²
}

O objectivo do presente estudo foi o de avaliar o impacto de duas dimensões específicas do clima de equipa (clima de orientação para a aprendizagem e clima de segurança psicológica), enquanto factor contextual de natureza cognitiva, nos diferentes tipos de conflito das equipas. Colocou-se a hipótese de que a relação entre orientação para a aprendizagem e os diferentes tipos de conflito é moderada pelo clima de segurança psicológica. Participaram neste estudo 218 equipas (959 indivíduos) envolvidas numa competição de estratégia e gestão que decorreu ao longo de cinco semanas. Globalmente, os resultados suportaram as hipóteses da influência directa do clima de equipa nos conflitos intragrupais no sentido em que climas mais positivos diminuem os conflitos nas equipas. Os resultados suportaram igualmente a hipótese do papel moderador da segurança psicológica na relação entre orientação para a aprendizagem e o conflito relacional e o conflito de gestão de tempo, sendo que a orientação para a aprendizagem reduz os conflitos quer relacionais quer de gestão de tempo nas situações de elevada segurança psicológica. Estes resultados chamam a atenção para a importância dos climas de equipa nos processos e dinâmicas entre os membros.

Agradecimentos:

Parte deste trabalho foi realizado no âmbito de um projecto de investigação financiado pela Fundação para a Ciência e Tecnologia (PTDC/PSI-PTO/102373/2008) e teve apoio do projecto PEst-OE/EGE/Ulo315/2011.

PALAVRAS-CHAVE: Segurança psicológica, orientação para a aprendizagem, conflito intragrupal, trabalho em equipa.

\section{Introdução}

Nos últimos anos assistimos a um interesse crescente pelo estudo da eficácia do trabalho em equipa em contexto organizacional. Este interesse deve-se, em

\footnotetext{
1 Instituto Universitário de Lisboa (ISCTE-IUL) - ana.passos@iscte.pt

2 Instituto Universitário de Lisboa (ISCTE-IUL), BRU-IUL, Lisboa.
} 
parte, ao facto de muitas organizações terem vindo a adoptar equipas de trabalho (como é o caso das equipas de projecto) como forma de garantir a flexibilidade e os níveis de eficácia exigidos nos contextos dinâmicos e competitivos em que estão inseridas.

Vários autores apontam a aprendizagem das equipas como um processo central que potencia a adaptação eficaz das organizações no contexto em que actuam (e.g., Decuyper, Dochy, \& Bossche, 2010; Kozlowski \& Bell, 2008; Zaccaro, Ely, \& Shuffler, 2008). De acordo com Edmondson (1999) a aprendizagem das equipas é "um processo contínuo de reflexão e acção caracterizado por colocar questões, procurar feedback, experimentar, reflectir sobre os resultados e discutir erros ou resultados inesperados das acções" (p. 353).

Apesar das equipas serem muitas vezes vistas como uma unidade de aprendizagem (Kozlowski \& Bell, 2003), é importante reconhecer que a mera reestruturação do trabalho tendo por base as equipas e não os indivíduos isoladamente, não garante os níveis de aprendizagem, adaptação e eficácia exigidos no actual contexto. Neste sentido, encontramos na literatura diversas propostas que procuram identificar as condições e as dinâmicas da equipa que promovem a aprendizagem.

A investigação tem-se centrado essencialmente nos resultados da aprendizagem das equipas dando especial atenção ao conhecimento e estruturas cognitivas que derivam da aprendizagem enquanto processo psicológico (Bell \& Kozlowski, 2008). Exemplo disso são os estudos realizados sobre o conhecimento colectivo (Ellis et al., 2003), os modelos mentais das equipas (Mohammed, Ferzandi, \& Hamilton, 2010) e a memória transactiva (Wegner, 1995). Por outro lado, alguns autores têm procurado igualmente estudar em que medida a aprendizagem das equipas se pode reflectir quer nos estados emergentes das equipas (Marks, Mathieu, \& Zaccaro, 2001) quer nos processos e dinâmicas que têm lugar nos episódios de tarefa levados a cabo pelos membros (Mathieu, Heffner, Goodwin, Salas, \& Cannon-Bowers, 2000).

Apesar dos desenvolvimentos recentes na literatura sobre o processo de aprendizagem das equipas consideramos que persistem questões em aberto que justificam a pertinência deste estudo. Em primeiro lugar, o número de estudos que se foca nos preditores do próprio processo de aprendizagem, como é o caso do clima de equipa, é ainda reduzido. De facto, apesar da relevância do clima de equipa para as dinâmicas sociais entre os membros das equipas (Anderson \& West, 1998), a maioria dos estudos empíricos centra-se na relação entre clima de equipa e desempenho (e.g., Bunderson \& Sutcliffe, 2003; Van der Vegt \& Bunderson, 2005) descuidando a relação entre clima de equipa para a aprendizagem e os processos de equipa. Em segundo lugar, sendo o conflito intragrupal um processo presente 
na maioria das equipas de trabalho com implicações relevantes para o desempenho e qualidade das relações entre os membros, interessa compreender a relação entre clima de equipa e as diferentes formas que o conflito pode assumir. Por fim, a maioria dos estudos empíricos realizados sobre a aprendizagem das equipas adopta uma perspectiva sincrónica, ignorando as dinâmicas que se estabelecem ao longo do tempo. Neste sentido é fundamental adoptar desenhos de estudos mais complexos que permitam ultrapassar algumas das limitações apontadas na literatura neste domínio.

O objectivo do presente estudo é avaliar o impacto de duas dimensões específicas do clima de equipa (clima de orientação para a aprendizagem e clima de segurança psicológica), enquanto factor contextual de natureza cognitiva, nos diferentes tipos de conflito das equipas adoptando um desenho longitudinal.

\section{Orientação para a aprendizagem e os conflitos nas equipas}

A investigação tem vindo a demonstrar que o clima de equipa pode promover o desenvolvimento de objectivos e comportamentos de aprendizagem entre os seus membros (Bunderson \& Sutcliffe, 2003; Edmondson, 1999) sendo, portanto, um preditor importante do próprio processo de aprendizagem. Neste sentido, o clima de equipa funciona como um factor contextual de natureza cognitiva que promove a aprendizagem da equipa através do seu impacto no funcionamento e dinâmicas entre os membros das equipas (Kozlowski \& Bell, 2008).

O clima de equipa pode ser definido como a percepção partilhada entre os membros de uma equipa sobre diversos aspectos relacionados com o seu funcionamento e contexto de trabalho (Anderson \& West, 1998). Tal como West e Richter (2011) mencionam, o clima de equipa é "what it feel like to work on a given team" (p.250).

Os resultados das investigações levadas a cabo nas últimas décadas sugerem que o clima de equipa está associado a uma variedade de processos e resultados do trabalho em equipa. Por exemplo, o clima de equipa é preditor da qualidade dos cuidados prestados ao nível da saúde (Edmondson, 1996), da satisfação, da produtividade e inovação (West \& Borrill, 2005) assim como dos comportamentos de segurança (Silva, 2008). No que diz respeito ao clima de aprendizagem, os poucos estudos realizados sugerem que este tipo específico de clima tem igualmente implicações importantes para as equipas de trabalho (Anderson \& West, 1998). Bunderson e Sutcliffe (2003) avaliaram a relação entre orientação para a aprendizagem (definida como o clima de equipa para a aprendizagem proactiva e o desenvolvimento de competências entre os membros) e o desempenho objectivo 
de um conjunto de unidades de negócio. Os resultados do estudo sugerem que existe uma relação positiva entre a orientação para a aprendizagem e a eficácia do trabalho em equipa. Segundo os autores, o estudo da aprendizagem da equipa deve ir para além do como as equipas aprendem (ou seja, o estudo dos processos de equipa associados à aprendizagem) para incluir igualmente questões relacionadas com a ênfase que os membros colocam na própria aprendizagem.

Modelos recentes sobre aprendizagem nas equipas sugerem que o conflito constitui um processo básico do próprio processo de aprendizagem. Contudo, a relação entre orientação para a aprendizagem e os conflitos nas equipas não tem sido alvo de investigação. Decuyper et al. (2010) consideram que o conflito pode ser entendido como um processo de negociação e diálogo que tem as suas origens na diversidade entre os membros. Através da interacção, o conflito permite a integração e a co-construção de significados comuns entre os membros e, portanto, a aprendizagem das equipas.

No seu modelo de aprendizagem das equipas, Decuyper et al. (2010) focam-se exclusivamente no papel do conflito construtivo para a aprendizagem das equipas. Contudo, na literatura é possível identificar diferentes tipos de conflito, nomeadamente o conflito de tarefa, o conflito relacional (e.g., Jehn, 1995; Jehn, 1997; Jehn \& Chatman, 2000; Jehn \& Mannix, 2001) e mais recentemente o conflito de gestão de tempo (Mohammed, Hamilton \& Lim (2009); Standifer et al., 2011). O conflito de tarefa caracteriza-se pela consciência de que existe desacordo entre os membros ao nível das ideias, das opiniões e dos pontos de vista sobre a tarefa a realizar pela equipa (Jehn, 1995; Jehn \& Mannix, 2001). O conflito relacional diz respeito à percepção de divergências baseadas em assuntos pessoais e sociais que não se relacionam com a tarefa em si mesma e envolvem sentimentos de frustração, de irritação e de tensão entre os membros (Jehn, 1995; Jehn \& Chatman, 2000). Por último, o conflito de gestão de tempo pode ser entendido como um tipo específico de conflito de processo (Jehn, 1997) e caracteriza-se pela divergência de opiniões sobre a forma como utilizar o tempo durante a realização do trabalho (Standifer et al., 2011).

Conceptualmente, a literatura sugere que enquanto o conflito de tarefa, também designado de conflito cognitivo, é funcional com impacto positivo no desempenho, especialmente em equipas cuja tarefa envolve a tomada de decisão (De Dreu, 1997; Jehn, 1995), o conflito relacional, ou conflito emocional, é prejudicial, quer para o desempenho quer para as respostas afectivas dos membros da equipa (Amason, 1996; Simons \& Peterson, 2000). No que se refere ao impacto do conflito de gestão de tempo no desempenho e respostas afectivas das equipas, o número de estudos empíricos que investigam directamente este tipo de conflito ainda é em número muito reduzido. Contudo, este tipo de conflito pode impossibilitar a equipa de 
coordenar eficazmente as acções individuais, afectando negativamente quer o desempenho quer as respostas afectivas dos membros (Standifer et al., 2011).

Tal como já foi referido anteriormente, a relação entre orientação para a aprendizagem e os conflitos nas equipas não tem sido explorada na literatura. A maioria dos estudos realizados sobre os preditores do conflito intragrupal centra-se essencialmente nas variáveis de composição das equipas, como é o caso da diversidade (e.g., Pelled, Eisenhardt, \& Xin, 1999), nas características das tarefas a realizar (e.g., Pelled, 1996) e mais recentemente nas cognições partilhadas pelos membros (e.g, Santos \& Passos, 2010). Contudo, tal como Bunderson e Sutcliffe (2003) sugerem, o clima de orientação para a aprendizagem, mais do que as questões demográficas da equipa ou os aspectos estruturais da tarefa, pode promover os processos necessários à aprendizagem das equipas, como é o caso dos conflitos construtivos. Neste sentido, as equipas com elevada orientação para a aprendizagem tendem a encorajar uma maior compreensão cognitiva das questões que são discutidas pelos membros, promovendo o conflito de tarefa. Por outro lado, a orientação para a aprendizagem tende a reduzir quer os conflitos relacionais quer os conflitos de gestão de tempo. Um clima de equipa que enfatiza o desenvolvimento de conhecimentos, de competências e do desempenho leva a que os membros estejam mais focados na tarefa a realizar do que nos seus interlocutores, ao mesmo tempo que tende a reduzir as atribuições negativas sobre os comportamentos levados a cabo pelos membros na concretização e na gestão das suas actividades.

Assim, tendo por base os pressupostos teóricos é esperado que:

H1a: A orientação para a aprendizagem aumenta os conflitos de tarefa na equipa. H1b: A orientação para a aprendizagem reduz os conflitos relacionais na equipa. H1c: A orientação para a aprendizagem reduz os conflitos de gestão de tempo na equipa.

\section{O papel da segurança psicológica}

A colaboração e a crítica construtiva nas equipas são possivelmente dos maiores desafios que os membros, os líderes e as organizações enfrentam nas suas actividades diárias. Se é consensual que a colaboração é essencial à aprendizagem e à eficácia das equipas e das organizações em geral, a conjugação de interesses distintos através da interacção, a partilha de informação e a coordenação das actividades entre os membros constitui, sem qualquer dúvida, uma área crítica que deve ser alvo de investigação e reflexão (Edmondson \& Roloff, 2009). 
A segurança psicológica pode ser definida como um "clima de equipa caracterizado pela confiança e respeito mútuo no qual as pessoas se sentem confortáveis a serem elas próprias" (Edmondson, 1999, p. 354). Vários autores sugerem que a segurança psicológica é facilitadora do próprio processo de aprendizagem nas equipas (e.g., Decuyper et al., 2010; Edmondson, 1999) uma vez que permite aos membros diminuírem a sua preocupação excessiva com as reacções dos outros a comportamentos e acções que, em outras circunstâncias, podem ser entendidos como ameaçadores à própria equipa.

A existência de um clima de segurança psicológica tem sido apontada como uma possivel variável moderadora na relação entre objectivos de aprendizagem e processos de aprendizagem de equipa (Edmondson, 2003) e na relação entre inovação e desempenho (Baer \& Frese, 2003). Nomeadamente, tem sido sugerido que um clima caracterizado por uma maior segurança psicológica irá funcionar como um facilitador de aprendizagem, discussão e inovação e irá mitigar os riscos interpessoais associados à aprendizagem.

A relação entre segurança psicológica e conflitos nas equipas tem sido estranhamente negligenciada na literatura. Contudo, alguns estudos sugerem indirectamente que um clima caracterizado por elevada segurança psicológica tende a diminuir os conflitos disfuncionais nas equipas, evitando simultaneamente a contaminação entre os diferentes tipos de conflito (De Dreu \& Weingart, 2003). Por exemplo, Simons e Peterson (2000) verificaram que a confiança nas equipas é moderadora da relação entre conflito de tarefa e conflito relacional. Segundo os autores, a confiança pode ser vista como uma variável chave que permite à equipa maximizar os benefícios do conflito de tarefa sem os custos associados ao conflito relacional. Edmondson e Roloff (2009) consideram que a segurança psicológica pode funcionar como uma variável de contexto moderando a relação entre a composição das equipas e os comportamentos de aprendizagem. De acordo com os autores, a segurança psicológica cria um contexto no qual é permitido a discussão explícita das diferenças de opiniões e pontos de vista por parte dos membros. Na mesma linha, Lau e Murnighan (2005) sugerem que a segurança psicológica reduz os efeitos negativos das faultines, enquanto medida de diversidade, nos conflitos intragrupais.

Decorrente da revisão de literatura, colocamos as seguintes hipóteses referentes ao papel da segurança psicológica nos conflitos intragrupais:

H2a: A segurança psicológica aumenta os conflitos de tarefa na equipa.

H2b: A segurança psicológica reduz os conflitos relacionais na equipa.

H2c: A segurança psicológica reduz os conflitos de gestão de tempo na equipa. 
H3: A segurança psicológica é moderadora da relação entre orientação para a aprendizagem e o conflito nas equipas de tal forma que os efeitos da orientação para a aprendizagem no conflito (positivos ou negativos) serão maiores em condições de elevada segurança psicológica.

Para testar o modelo proposto sobre a relação entre a orientação para a aprendizagem e a segurança psicológica nos conflitos intragrupais, realizou-se um estudo empírico ao longo do tempo, tendo como base o nível de análise da equipa.

\section{Método}

\section{Participantes}

Neste estudo participaram 218 equipas (959 indivíduos) envolvidas numa competição de estratégia e gestão que decorreu ao longo de cinco semanas. As equipas tinham, em média, 4,56 elementos (D.P. = 0,69) e eram constituídas por estudantes, quadros de empresas ou mistas. Os participantes tinham, em média, 29 anos (D.P. $=8,67)$ e $69 \%$ eram do sexo masculino.

\section{Procedimento}

A competição de gestão consistiu numa simulação empresarial em que cada equipa gere uma empresa virtual. As equipas representavam empresas que competiam no mercado com vista a atingir a cotação mais elevada das suas acções na bolsa de valores. As equipas geriram as empresas através de tomadas de decisão semanais nas áreas de marketing, produção, recursos humanos e finanças. Um simulador analisou as decisões semanais de cada equipa, calculou o valor das acções de cada empresa e classificou cada equipa. Durante a competição, as equipas receberam feedback sobre a sua classificação, assim como das restantes equipas.

Durante a competição, os membros das equipas responderam a dois questionários on-line. O link de cada questionário foi enviado na segunda semana (Questionário 1) e na quarta semana (Questionário 2), para os membros da equipa, através de e-mail, os quais respondiam antes de receberem feedback sobre as decisões.

\section{Operacionalização das variáveis}

Segurança psicológica. A segurança psicológica foi avaliada através de dois itens adaptados da escala de Edmondson (1999). Os participantes indicavam o seu grau 
de acordo com cada uma das afirmações ("Os membros da minha equipa não toleram os erros uns dos outros" e "Na minha equipa é difícil pedir auxílio aos outros membros da equipa"), numa escala tipo Likert de 7 pontos ( 1 = Discordo totalmente a $7=$ Concordo totalmente). Na análise dos resultados a escala foi invertida, o que significa que valores mais baixos correspondem a baixa segurança psicológica e valores mais altos a elevada segurança psicológica. A segurança psicológica foi avaliada no questionário 1.

Orientação da equipa para a aprendizagem. Foram adaptados dois itens da escala de Bunderson e Sutcliffe (2003) para avaliar a orientação da equipa para a aprendizagem. Os participantes indicavam o seu grau de acordo com as afirmações ("Os membros da minha equipa procuram aprender e desenvolver as suas competências" e "Os membros da minha equipa estão dispostos a arriscar em novas ideias de modo a descobrir o que funciona melhor") numa escala tipo Likert de 7 pontos (1 $=$ Discordo totalmente a $7=$ Concordo totalmente). A orientação da equipa para aprendizagem foi avaliada no questionário 1.

Conflito intragrupal. O conflito de tarefa e o conflito relacional foram operacionalizados através da Escala de Conflito Intragrupal de Jehn $(1995,1997)$. O conflito de gestão de tempo foi operacionalizado através da escala de Standifer et al. (2011). Os participantes indicavam a frequência com que ocorreram determinadas situações na sua equipa, durante a competição, numa escala tipo Likert de 7 pontos ( 1 = Nunca a $7=$ Sempre). A escala do conflito era constituída por 11 itens, sendo quatro do conflito de tarefa (e.g., "Os membros manifestaram desacordo acerca do conteúdo das decisões”), quatro do conflito relacional (e.g., "Os membros são postos em causa por expressarem as suas opiniões pessoais") e três do conflito de gestão de tempo (e.g., "Existe desacordo entre os membros sobre a forma de distribuir o tempo disponível na realização de tarefas"). O conflito intragrupal foi avaliado no questionário 2.

\section{Resultados}

\section{Agregação}

Uma vez que o nivel de análise neste estudo foi a equipa, as respostas individuais foram agregadas para o nivel de equipa para posterior análise. De forma a justificar a agregação, avaliámos o acordo entre equipas, através do cálculo $R_{w g(j)}$ (James, Demaree, \& Wolf, 1993), bem como a fiabilidade e consistência através dos cálculos das correlações intraclasses (Bliese, 2000). Todos os valores estavam de acordo com os critérios exigidos (ver Quadro 1) e, por isso, as respostas individuais foram agregadas para o nível de equipa. 


\section{Teste de hipóteses}

No Quadro 1 podem ser consultadas as médias, desvios-padrão e correlações de todas as variáveis do estudo, a nível da equipa. Como esperado, os resultados revelam correlações significativas entre todas as variáveis. As variáveis preditoras segurança psicológica e orientação para a aprendizagem correlacionaram-se de forma positiva e significativa entre si $(r=.51, p<.01)$ e correlacionaram-se de forma negativa e significativa com as variáveis critério.

Quadro 1. Estatísticas descritivas e correlações das variáveis em estudo

\begin{tabular}{|c|c|c|c|c|c|c|c|c|c|c|}
\hline & $\operatorname{ICC}(1)$ & $\operatorname{ICC}(2)$ & $\operatorname{Rwg}(\mathrm{j})$ & M & SD & 1 & 2 & 3 & 4 & 5 \\
\hline $\begin{array}{l}\text { 1. Segurança } \\
\text { psicológica }\end{array}$ & .11 & .37 & .72 & 5.93 & .81 & $(.53) 1$ & & & & \\
\hline $\begin{array}{l}\text { 2. Orientação } \\
\text { aprendizagem }\end{array}$ & .07 & .26 & .80 & 5.71 & .62 & .51 & $(.74) 1$ & & & \\
\hline 3. Conflito tarefa & .11 & .37 & .73 & 2.95 & .83 & -.28 & -.26 & $(.82) 2$ & & \\
\hline $\begin{array}{l}\text { 4. Conflito } \\
\text { relacional }\end{array}$ & .23 & .57 & .79 & 1.77 & .89 & -.54 & -.48 & .55 & $(.93)^{2}$ & \\
\hline $\begin{array}{l}\text { 5. Conflito gestão } \\
\text { de tempo }\end{array}$ & .14 & .43 & .72 & 2.20 & .84 & -.44 & -.43 & .67 & .83 & $(.91) 2$ \\
\hline
\end{tabular}

Nota. $N=218$ equipas

Todas as correlações são estatisticamente significativas $\operatorname{com} p<.01$.

Os coeficientes de fidelidade são apresentados na diagonal, entre parênteses.

'Coeficientes de correlação de Pearson

${ }^{2}$ Alfas de Cronbach

Para avaliar as hipóteses em estudo realizámos Regressões Múltiplas Hierárquicas, sendo que para os modelos de moderação recorremos ao método ENTER. Assim, os efeitos principais das variáveis entraram no primeiro passo do modelo, e os efeitos de interacção no segundo passo. As variáveis preditoras foram centradas previamente, de acordo com o procedimento proposto por Aiken e West (1991).

No que respeita aos efeitos directos, os resultados mostraram efeitos significativos e negativos entre as duas variáveis preditoras e todas as variáveis critério (ver Quadro 2). Neste sentido, verificou-se um efeito principal negativo e significativo da orientação para a aprendizagem e da segurança psicológica no conflito de tarefa ( $B$ $=-.21, p<.05$ e $B=-.21, p<.01$, respectivamente). Este modelo explica $9 \%$ de variância $(F=11.06, p<.001)$. Estes resultados não permitiram suportar a Hipótese 1a e 2 .

No que concerne ao conflito relacional, os resultados mostraram que a orientação para a aprendizagem tem um efeito principal negativo e significativo no mesmo 
( $B=-.40, p<.01)$, assim como a segurança psicológica $(B=-.44, p<.001)$ explicando $34 \%$ de variância $(F=54.47, p<.001)$. Estes resultados permitem suportar as Hipóteses $1 b$ e $2 b$.

Por fim, como esperado, verificou-se um efeito negativo e significativo da orientação para a aprendizagem e da segurança psicológica no conflito de gestão de tempo ( $B=-.38, p<.001$ e $B=-.31, p<.001$, respectivamente). Este modelo explica $24 \%$ de variância $(F=34.16, p<.001)$. Os resultados permitem suportar as Hipóteses $1 c$ e $2 c$.

Relativamente aos efeitos de moderação da segurança psicológica na relação entre a orientação para a aprendizagem e o conflito de tarefa, os resultados revelaram que quando a interacção entre as duas variáveis preditoras foi adicionada à equação, a interacção não foi estatisticamente significativa, assim como o $F$ da mudança (ver Quadro 2). Assim, os resultados não permitem suportar a Hipótese 3 , no que respeita aos conflitos de tarefa.

Quadro 2. Resultados das regressões múltiplas hierárquicas dos modelos de moderação

\begin{tabular}{|c|c|c|c|c|c|c|}
\hline \multirow[b]{2}{*}{ Modelo } & \multicolumn{2}{|c|}{ Conflito tarefa } & \multicolumn{2}{|c|}{ Conflito relacional } & \multicolumn{2}{|c|}{$\begin{array}{l}\text { Conflito gestão de } \\
\text { tempo }\end{array}$} \\
\hline & Passo 1 & Passo 2 & Passo 1 & Passo 2 & Passo 1 & Passo 2 \\
\hline \multicolumn{7}{|l|}{$\begin{array}{l}\text { 1. Efeitos } \\
\text { principais }\end{array}$} \\
\hline $\begin{array}{l}\text { Orientação } \\
\text { aprendizagem }\end{array}$ & $-.21^{*}(.10)$ & $-.21^{*}(.11)$ & $-.40^{* *}(.09)$ & $-.32^{* *}(.09)$ & $-.38^{* * *}(.10)$ & $-.35^{* * *}(.10)$ \\
\hline $\begin{array}{l}\text { Segurança } \\
\text { psicológica }\end{array}$ & $-.21^{* *}(.08)$ & $-.21^{* *}(.08)$ & $-.44^{* * *}(.07)$ & $-.34^{* * *}(.07)$ & $-.31^{* * *}(.07)$ & $-.27^{* * *}(.08)$ \\
\hline \multicolumn{7}{|l|}{ 2. Interacção } \\
\hline $\begin{array}{l}\text { Orientação } \\
\text { aprendizagem } \\
\text { x Segurança } \\
\text { psicológica }\end{array}$ & & $.01(.09)$ & & $-.36^{* * *}(.08)$ & & $-.16+(.08)$ \\
\hline Adj. R2 & .09 & .08 & .34 & .39 & .24 & .25 \\
\hline$\Delta R_{2}$ & & .00 & & $.06^{* * *}$ & & $.01 \dagger$ \\
\hline $\mathrm{F}$ & $11.06^{* * *}$ & $7.35^{* * *}$ & $54.47^{* * *}$ & $46.58^{* * *}$ & $34.16^{* * *}$ & $24.25^{* * *}$ \\
\hline$\Delta \mathrm{F}$ & & 3.71 & & $7.90^{* * *}$ & & $9 \cdot 91^{* * *}$ \\
\hline
\end{tabular}

Nota. São apresentados os betas não-estandardizados

Os valores dos erros estandardizados são apresentados entre parênteses

${ }^{\dagger}=.06 ;{ }^{*} p<.05 ;{ }^{* *} p<.01 ;{ }^{* * *} p<.001$

No que respeita ao conflito relacional, quando a interacção entre a orientação para a aprendizagem e a segurança psicológica foi adicionada à equação, a interacção foi estatisticamente significativa ( $B=-.36, p<.001$ ), tal como o $F$ da mudança (ver Quadro 2). Estes resultados reflectem uma possível moderação. 
Para testar a moderação, com base nos procedimentos sugeridos por Aiken e West (1991), realizámos os coeficientes da equação de regressão (b) de forma a reflectirem os diferentes níveis de moderação. Calculámos um desvio-padrão acima e um desvio-padrão abaixo da média de forma a representarem níveis de segurança psicológica elevados e baixos, respectivamente. A Figura 1 mostra o efeito de interacção da orientação para a aprendizagem e da segurança psicológica no conflito relacional. É possível verificar que o efeito da orientação para a aprendizagem no conflito relacional é negativo e significativo na condição baixa segurança psicológica $(B=-.61 ; t=-6.03 ; p<.001)$. Em sentido contrário, o efeito da orientação para a aprendizagem no conflito relacional é negativo e nãosignificativo na condição elevada segurança psicológica ( $B=-.02 ; t=-.20 ; p=.85$ ). Estes resultados suportam a Hipótese 3 , no que respeita aos conflitos relacionais.

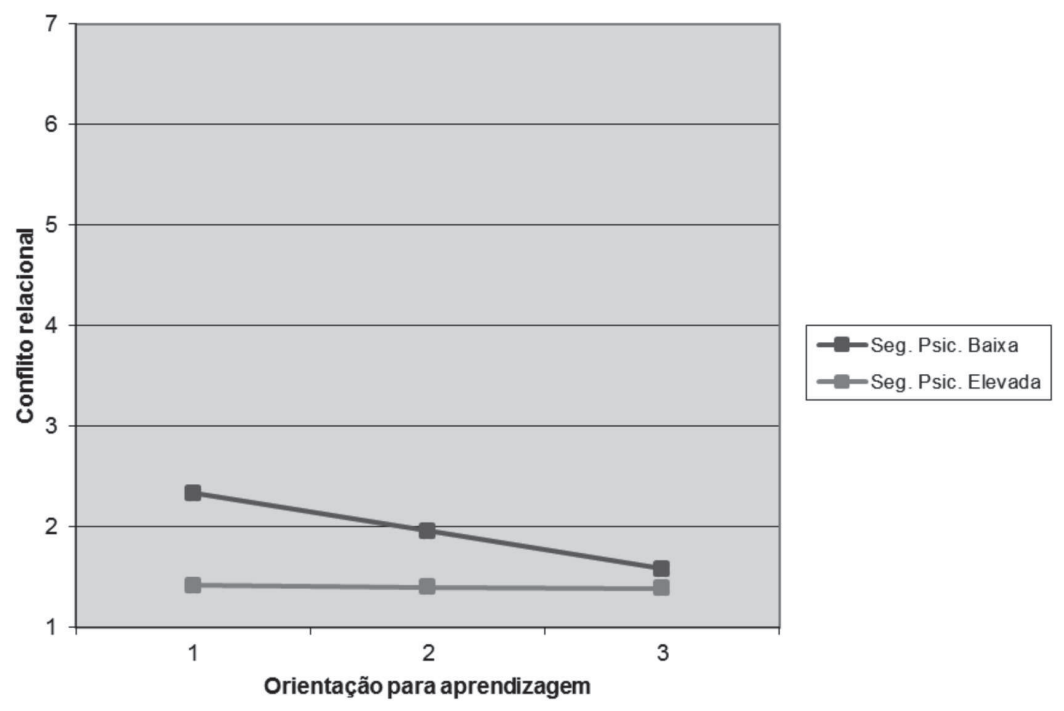

Figura 1. Moderação da segurança psicológica entre a orientação para a aprendizagem e o conflito relacional.

Finalmente, no que respeita ao conflito de gestão de tempo, quando adicionada à equação, a interacção entre a orientação para a aprendizagem e a segurança psicológica teve um pequeno efeito significativo e negativo ( $B=-.16, p=.06)$. 0 $F$ da mudança foi estatisticamente significativo (ver Quadro 2). Estes resultados reflectem uma possivel moderação da segurança psicológica.

Para testar a moderação, seguimos os procedimentos descritos anteriormente. A Figura 2 mostra o efeito de interacção da orientação para a aprendizagem e da segurança psicológica no conflito de gestão de tempo. É possível verificar que o efeito da orientação para a aprendizagem no conflito de gestão de tempo é nega- 
tivo e significativo em situações de baixo nível de segurança psicológica ( $B=-.48$; $t=-4.45 ; p$ <.001). Contrariamente, o efeito da orientação para a aprendizagem no conflito de gestão de tempo é negativo e não-significativo em situações de elevado nível de segurança psicológica ( $B=-.22 ; t=-1.68 ; p=.09$ ). Assim, os resultados suportam a Hipótese 3 , no que respeita aos conflitos de gestão de tempo.

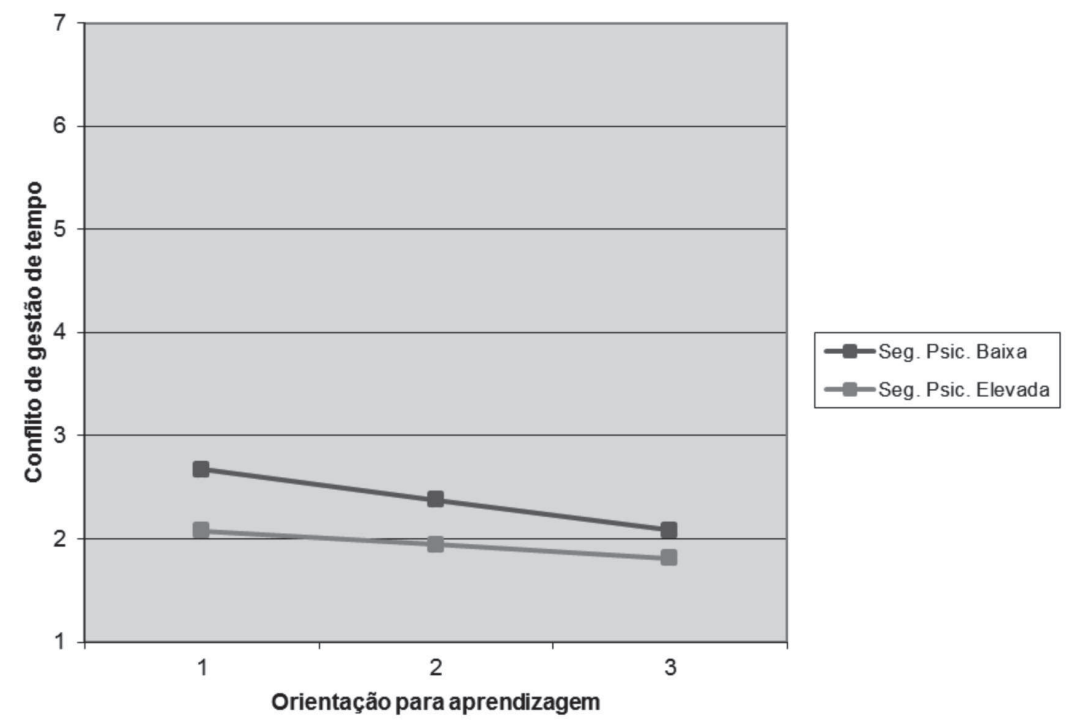

Figura 2. Moderação da segurança psicológica entre a orientação para a aprendizagem e o conflito de gestão de tempo.

\section{Discussão}

Este estudo teve como principal objectivo avaliar o papel da orientação para a aprendizagem e da segurança psicológica nos diferentes tipos de conflito intragrupal. Globalmente, os resultados suportaram as hipóteses do estudo quer da influência directa da orientação para a aprendizagem e da segurança psicológica nos conflitos, quer do efeito de moderação da segurança psicológica.

Em relação à influência directa da orientação para a aprendizagem e da segurança psicológica no conflito de tarefa, os resultados não permitiram suportar as hipóteses $1 a$ e 2 a. Ao contrário do esperado, tendo por base a perspectiva da controvérsia construtiva (Decuyper et al., 2010), a influência destes dois tipos de clima reduz significativamente os conflitos cognitivos nas equipas. Os resultados encontrados na literatura relativos ao conflito de tarefa têm-se revelado muito inconsistentes quer ao nível dos seus preditores quer ao nível das suas consequências. Numa meta- 
análise à literatura sobre conflito intragrupal, De Dreu e Weingart (2003) analisam as associações encontradas nas investigações empíricas entre conflito de tarefa e conflito relacional, por um lado, e resultados do trabalho em equipa (desempenho das equipas e satisfação dos membros). Em relação aos efeitos do conflito intragrupal no desempenho das equipas, os resultados mostram que tanto o conflito de tarefa como o conflito relacional são prejudiciais. Apesar da controvérsia construtiva através da discussão de ideias e de pontos de vista aumentar potencialmente a aprendizagem das equipas (Woerkom \& Sanders, 2010), os resultados do presente estudo sugerem que em climas de elevada orientação para a aprendizagem o desacordo, mesmo ao nível cognitivo, é provavelmente interpretado como negativo. Este resultado vai de encontro ao de Schwenk (1990) que verificou que os gestores tendem a percepcionar o conflito como algo negativo, mesmo quando objectivamente o resultado final da tomada de decisão beneficia da discussão entre os membros. No mesmo sentido, Passos e Caetano (2005) verificaram que o conflito de tarefa diminui a percepção de eficácia das equipas sugerindo que o conflito pode ser entendido como um processo que distrai as equipas de atingirem o seu objectivo central. Por outro lado, podemos igualmente pressupor que em climas de equipa mais positivos, os processos de discussão e de desacordo entre os membros são interpretados como naturais e não conotados ou entendidos como conflitos.

No que diz respeito à influência directa da orientação para a aprendizagem e da segurança psicológica nos conflitos relacionais e nos conflitos de gestão de tempo os resultados permitiram suportar as hipóteses (hipóteses $1 b, 1 c, 2 b$ e $2 c$ ). De facto, as duas dimensões do clima reduzem significativamente a percepção quer dos conflitos relacionais quer dos conflitos de gestão de tempo entre os membros das equipas. Estes resultados sugerem que as equipas com elevada orientação para a aprendizagem estão mais focalizadas na tarefa que têm de realizar e nas actividades relacionadas com a concretização dos objectivos propostos, o que vai de encontro à proposta de Bunderson e Sutcliffe (2003). Da mesma forma, tal como era esperado, um clima de equipa caracterizado por uma elevada segurança psicológica diminui quer as tensões interpessoais entre os membros das equipas quer os desacordos relativos à gestão do tempo e das actividades a realizar. A elevada segurança psicológica nas equipas parece criar o contexto necessário para a troca aberta de ideias, evitando que os membros interpretem os desacordos como ataques pessoais. Estes resultados estendem a proposta inicial de Edmondson (1999) ao sugerirem que os climas positivos nas equipas têm impacto directo nos processos que caracterizam as dinâmicas do funcionamento das equipas, como é o caso do conflito intragrupal, e não apenas nos resultados finais do seu trabalho como tem vindo a ser demonstrado na literatura (e.g., Carmeli \& Gittell, 2009; Edmondson, 1999).

Os resultados do presente estudo permitiram suportar parcialmente a hipótese de moderação da segurança psicológica na relação entre orientação para a 
aprendizagem e o conflito intragrupal (hipótese 3). De facto, esta moderação não se verificou para o conflito de tarefa. No que diz respeito ao conflito relacional, os resultados mostram que em equipas com um clima de segurança psicológica elevado, a orientação para a aprendizagem não tem impacto nos atritos interpessoais que caracterizam os conflitos de natureza mais emocional. O conflito relacional parece surgir essencialmente em equipas com baixa orientação para a aprendizagem e baixa segurança psicológica. Estes resultados vão de encontro aos estudos empíricos realizados no âmbito da aprendizagem das equipas (e.g., Baer \& Frese, 2003) e às proposições teóricas recentemente propostas na literatura de Edmondson e Roloff (2009) do papel moderador do clima de segurança psicológica. De facto, a segurança psicológica parece criar um contexto no qual a discussão das ideias e pontos de vista distintos não coloca em causa os membros individualmente, facilitando o processo de aprendizagem nas equipas.

No que diz respeito ao conflito de gestão de tempo, os resultados permitiram igualmente suportar a hipótese de moderação da segurança psicológica entre a orientação para a aprendizagem e este tipo específico de conflito intragrupal. Tal como se verificou em relação ao conflito relacional, nas equipas com elevada segurança psicológica, não existe relação entre a orientação para a aprendizagem e o conflito de gestão de tempo. Por outro lado, é nas equipas com baixa segurança psicológica e baixa orientação para a aprendizagem que o conflito de gestão de tempo está mais presente. Apesar da investigação sobre este tipo de conflito estar a dar os primeiros passos, os estudos empíricos sugerem que o conflito de gestão de tempo é disfuncional para as equipas, reduzindo o desempenho e as respostas afectivas dos membros (e.g., Standifer et al., 2011). Neste sentido, torna-se importante, em estudos futuros, investigar em que medida o conflito intragrupal é mediador da relação entre os climas de equipa e a eficácia do trabalho em equipa.

O presente estudo tem implicações relevantes quer para a literatura da aprendizagem das equipas quer para a dos conflitos. Em primeiro lugar, os resultados obtidos parecem sugerir que a redução dos conflitos disfuncionais nas equipas, que a literatura tem vindo a demonstrar que comprometem a eficácia das equipas (e.g.. De Dreu \& Weingart, 2003), depende do clima de orientação para a aprendizagem e do clima de segurança psicológica isoladamente mas também da combinação destes diferentes tipos de clima. Estes resultados chamam a atenção para a necessidade dos líderes estimularem não só a aprendizagem proactiva e o desenvolvimento das competências relevantes para a tarefa dos membros mas, simultaneamente, desenvolverem um conjunto de acções que promovam a confiança e o respeito mútuo entre os membros, criando deste modo um clima de prevenção dos conflitos disfuncionais. Em segundo lugar, os resultados sugerem que a segurança psicológica pode ser uma variável importante para compreender em que circunstâncias os desacordos ao nível da tarefa não se transformam em 
conflitos relacionais. Ou seja, os resultados do presente estudo sugerem que nas equipas com elevada segurança psicológica, os comportamentos ambíguos manifestados em situações de debate de ideias são certamente menos interpretados como inapropriados ou como ataques pessoais, o que vai de encontro à proposta de Simons e Peterson (2000) sobre o papel da confiança nas equipas.

Apesar dos dados do presente estudo terem sido recolhidos ao longo do tempo, as análises efectuadas não traduzem efectivamente a dimensão temporal. De acordo com Decuyper et al. (2010) a ausência da dimensão temporal dos estudos realizados sobre a aprendizagem das equipas constitui uma das principais limitações da investigação neste domínio. De facto, a maioria dos autores tende a ignorar a fase de desenvolvimento das equipas, assumindo que os diferentes inputs e estados emergentes têm a mesma importância independentemente do ciclo de tarefa em que as equipas se encontram. Neste estudo, a utilização de equipas envolvidas numa competição de gestão permitiu, em certa medida, garantir que o ciclo de tarefa fosse equivalente para todas as equipas. Contudo, estudos futuros deverão utilizar métodos de análise de dados que permitam ter em linha de conta o momento específico do tempo em que as dinâmicas internas das equipas ganham relevância. Torna-se igualmente pertinente em estudos futuros testar modelos mais complexos da influência do clima das equipas nos processos e resultados, quer ao nível do desempenho objectivo, quer ao nível das respostas afectivos dos membros.

Em conclusão, o objectivo deste estudo foi o de contribuir para a melhor compreensão da relação entre climas de equipa e conflitos intragrupais, enquanto processos chave da eficácia do trabalho em equipa. Globalmente os resultados suportaram o modelo em análise e chamam a atenção para a importância dos climas positivos nas dinâmicas e funcionamento das equipas.

\section{Referências}

Aiken, L., \& West, S. G. (1991). Multiple regression: Testing and interpreting interactions. Newbury Park, CA: Sage.

Amason, A. C. (1996). Distinguishing the effects of functional and dysfunctional conflict on strategic decision making: Resolving a paradox for top management teams. Academy of Management Journal, 39 (1), 123-148.

Anderson, N., \& West, M. A.(1998). Measuring climate for work group innovation: Development and validation of the Team Climate Inventory. Journal of Organizational Behavior, 19, 235- 258 .

Baer, M., \& Frese, M. (2003). Innovation is not enough: Climates for initiative and psychological safety, process innovations, and firm performance. Journal of Organizational Behavior, 24, 45-68. 
Bell, B. S., \& Kozlowski, S. W. J. (2008). Active learning: Effects of core training design elements on self-regulatory processes, learning, and adaptability. Journal of Applied Psychology, 93(2), 296-316.

Bliese, P. D. (2000).Within-group agreement, non-independence, and reliability: Implications for data aggregation and analysis. In K. J. Klein \& S.W.J.Kozlowski (Eds.), Multilevel Theory, Research, and Methods in Organizations (pp. 349-381). San Francisco: Jossey-Bass.

Bunderson, J.S., \& Sutcliffe, K.M. (2003). Management team learning orientation and business unit performance. Journal of Applied Psychology, 88(3), 552-560.

Carmeli, A., \& Gittell, J. H. (2009). High-quality relationships, psychological safety, and learning from failures in work organizations. Journal of Organizational Behavior, 30, 709-729.

De Dreu, C. K. W. (1997). Productive conflict: The importance of conflict issue and conflict management. In C. K.W. De Dreu, \& E. Van de Vliert (Eds.), Using conflict in organizations (pp. 9 - 22). London: Sage.

De Dreu, C. K. W., \& Weingart, L. R. (2003). Task versus relationship conflict, team performance, and team member satisfaction: A meta-analysis. Journal of Applied Psychology, 88, 741-749.

Decuyper, S., Dochy, F., \& Van den Bossche, P. (2010). Grasping the dynamic complexity of team learning: An integrative model for effective team learning in organizations. Educational Research Review, 5, 111-133.

Edmondson, A.C. (1996). Learning from mistakes is easier said than done: Group and organization influences on the detection and correction of human error. Journal of Applied Behavioral Science, 32 (1), 5-28.

Edmondson, A. (1999). Psychological safety and learning behavior in work teams. Administrative Science Quarterly, 44(2), 350-383.

Edmondson, A.C. (2003). Managing the risk of learning: Psychology safety in work teams. In M.West (Ed.), International handbook of organizational teamwork and cooperative working (pp. 255-276). London: Blackwell.

Edmondson, A. C., \& Roloff, K. S. (2009). Overcoming Barriers to Collaboration: Psychological Safety and Learning in Diverse Teams. In E. Salas, G. G. Goodwin, \& C. S. Burke (Eds.), Team Effectiveness in Complex Organizations: Cross-disciplinary Perspectives and Approaches (pp. 183-208). New York: Psychology Press.

Ellis, A. P. J., Hollenbeck, J. R., Ilgen, D. R., Porter, L. H., West, B. J., \& Moon, H. (2003). Team learning: Collectively connecting the dots. Journal of Applied Psychology, 88, 821-835.

James, L. R., Demaree, R. J., \& Wolf, G. (1993). Rwg: An assessment of within group interrater agreement. Journal of Applied Psychology, 78, 306-309.

Jehn, K. A. (1995). A multi method examination of the benefits and detriments of intragroup conflict. Administrative Science Quarterly, 40, 256-282.

Jehn, K.A. (1997). A qualitative analysis of conflict types and dimensions in organizational groups. Administrative Science Quarterly, 42, 530-557.

Jehn, K. A., \& Chatman, J. A. (2000). The influence of proportional and perceptual conflict composition on team performance. International Journal of Conflict Management, $11,56-73$.

Jehn, K. A., \& Mannix, E. (2001). The dynamic nature of conflict: A longitudinal study of intragroup conflict and group performance. Academy of Management Journal, 44, 238-251.

Kozlowski, S. W. J., \& Bell, B.S. (2008). Team learning, development, and adaptation. In V. I. Sessa \& M. London (Eds.), Group learning (pp.15-44). Mahwah, NJ: LEA 
Kozlowski, S. W. J., \& Bell, B. S. (2003). Work groups and teams in organizations. In W. C. Borman, D. R. Ilgen \& E. J. Klimoski (Eds.), Handbook of psychology: Industrial and organizational psychology (vol.12, pp. 333-375). London, UK: Wiley.

Lau, D. C., \& Murnighan, J. K. (2005). Interactions within Groups and Subgroups: The Effects of Demographic Faultlines. Academy of Management Journal, 48(4), 645-659.

Marks, M. A., Mathieu, J. E., \& Zaccaro, S. J. (2001). A temporally based framework and taxonomy of team processes. Academy of Management Review, 26, 356-376.

Mathieu, J. E., Heffner, T.S., Goodwin, G. F., Salas, E., \& Cannon-Bowers, J. A. (2000). The influence of shared mental models on team process and performance. Journal of Applied Psychology, 85, 273-283.

Mohammed, S., Ferzandi, L., \& Hamilton, K. (2010). Metaphor no more: A 15-year review of the team mental model construct. Journal of Management, 36 (4), 876-910.

Mohammed, S., Hamilton, K., \& Lim, A. (2009). The incorporation of time in team research: Past, current, and future. In E. Salas, G. F. Goodwin, C. S. Burke (Eds.), Team Effectiveness in Complex Organizations: Cross-Disciplinary Perspectives and Approaches (pp. 321-350). New York: Psychology Press.

Passos, A., \& Caetano, A. (2005). Exploring the effects of intragroup conflict and past performance feedback on team effectiveness. Journal of Managerial Psychology, 20, (3/4), 231-244.

Pelled, L. H. (1996). Demographic diversity, conflict, and work group outcomes: An intervening process theory, Organization Science, 7, 615-631.

Pelled, L. H., Eisenhardt, K. M., \& Xin, K. R. (1999). Exploring the black box: An analysis of work group diversity, conflict, and performance. Administrative Science Quarterly, 44(1), 1-28.

Santos, C., \& Passos, A. (2010). A influência dos modelos mentais de equipa na eficácia ao longo do tempo: uma relação mediada pelo conflito (Dissertação de Mestrado não publicada). Instituto Universitário de Lisboa (ISCTE-IUL), Lisboa.

Schwenk, C. R. (1990). Conflict in organizational decision making: An exploratory study of its effects in for-profit and not-for-profit organizations. Management Science, 36(4), 436-448.

Silva, S. (2008). Culturas de segurança e prevenção de acidentes de trabalho numa abordagem psicossocial: Valores organizacionais declarados e em uso. Lisboa: Fundação Calouste Gulbenkian.

Simons, T., \& Peterson, R. (2000). Task conflict and relationship conflict in top management teams: The pivotal role of intragroup trust. Journal of Applied Psychology, 85, 102-111.

Standifer, R. L., Raes, A. M. L., Peus, C., Passos, A. M., Santos, C., Weisweiler, S., \& Bruch, H. (2011). The relationships of shared temporal cognitions, temporal conflict, and team processes to team member satisfaction: A comparative study across four countries. Manuscript submitted for publication.

Van Der Vegt, G., \& Bunderson, J. S. (2005). Learning and performance in multidisciplinary teams: The importance of collective team identification. Academy of Management Journal, 48(3), 532-547.

Wegner, D. M. (1995). A computer network model of human transactive memory. Social Cognition, 13, 1-21.

West, M. A., \& Borrill, C. S. (2005). The influence of team working. In J. Cox, J. King, A. Hutchinson, \& P. McAvoy (Eds.), Understanding doctors' performance (pp. 106-122). Oxford: Radcliffe.

West, M., \& Richter, A. W. (2011). Team climate and effectiveness outcomes. In N. M. Ashkanasy, C. P. M. Wilderom, \& M. F. Peterson (Eds.), The Handbook of Organizational Culture and Climate (pp.249-270). Thousand Oaks: SAGE Publications. 
Woerkom, M., \& Sanders, K. (2010). The romance of learning from disagreement. The effect of cohesiveness and disagreement on knowledge sharing behavior and individual performance within teams. Journal of Bussiness Psychology, 25, 139-149.

Zaccaro, S. J., Ely, K., \& Shuffler, M. (2008). The leader's role in group learning. In V. Sessa, \& M. London (Eds.), Work group learning. Understanding, improving \& assessing how groups learn in organizations (pp. 15-44). Mahwah, NJ: Lawrence Erlbaum Associates.

\section{Effects of learning orientation and psychological safety on team conflict}

This study aimed to evaluate the impact of two specific dimensions of team climate (learning orientation and psychological safety), as a cognitive contextual factor, on different types of team conflict. We hypothesized that the relationship between learning orientation and the different types of conflict is moderated by psychological safety climate. A total of 218 teams (959 individuals) participated in this study. All teams were enrolled in a strategic and management competition for a 5-week period. Globally, the results supported the hypotheses concerning the direct influence of team climate on intragroup conflicts, since more positive climates diminish team conflict. The results also supported the hypothesis of the moderating role of psychological safety on the relationship between learning orientation and both relationship conflict and temporal conflict. Learning orientation diminishes both relationship and temporal conflicts when teams experience high levels of psychological safety. These results point out the importance of team climates on processes and dynamics among members.

KEY-WORDS: Psychological safety, learning orientation, intragroup conflict, team work 